\title{
INTERSECTION AND CLOSURE
}

\author{
W. H. GOTTSCHALK
}

In a topological space the closure of the union of two sets is the union of the closures. We here consider the question when the closure of the intersection of two sets is the intersection of the closures. We use the technique of uniform spaces. All uniform spaces mentioned are understood to be $T_{0}$-spaces. If $E$ is a set and if $\alpha$ is a vicinity, then $E \alpha$ denotes the $\alpha$-neighborhood of $E$.

Let $X$ be a \{topological\} \{uniform\} space and let $A, B \subset X$. The sets $A$ and $B$ are said to be $\{$ continuously\} \{uniformly\} separated provided there exists a \{continuous $\}$ uniformly continuous $\}$ map $\phi$ of $X$ into the unit interval such that $x \phi=0(x \in A)$ and $x \phi=1$ $(x \in B)$.

REMARK 1. Let $X$ be a uniformizable space, let $U$ be a compatible uniformity of $X$ which contains the beta-uniformity (that is, the greatest precompact compatible uniformity) of $X$, and let $A, B \subset X$. Then the following statements are pairwise equivalent:

(1) $A$ and $B$ are continuously separated.

(2) $A$ and $B$ are uniformly separated relative to $U$.

(3) $A$ and $B$ are uniformly separated relative to some compatible uniformity of $X$.

REMARK. 2. Let $X$ be a uniform space and let $A, B \subset X$. Then $A$ and $B$ are uniformly separated if and only if there exists a vicinity $\alpha$ of $X$ such that $A \alpha \cap B=\varnothing$. (Cf. [1].)

Remarks 1 and 2 may be taken as definitions.

Let $X$ be a $\{$ topological\} \{uniform\} space and let $A, B \subset X$. The sets $A$ and $B$ are said to be $\{$ continuously\} \{uniformly\} separable provided that if $U$ is a neighborhood of $A \cap B$ such that $A \cap B$ and $X-U$ are continuously\} \{uniformly\} separated, then $A-U$ and $B-U$ are $\{$ continuously\} \{uniformly\} separated.

REMARK 3. Remark 1 with "separated" replaced by "separable."

REMARK 4. Let $X$ be a uniform space and let $A, B \subset X$. Then:

(1) If $A$ is compact, if $B$ is closed, and if $A \cap B=\varnothing$, then $A$ and $B$ are uniformly separated.

(2) If $A$ is compact and if $B$ is closed, then $A$ and $B$ are uniformly separable.

Theorem 1. Let $X$ be a uniform space and let $A, B \subset X$. Then the

Presented to the Society, October 25, 1952; received by the editors August 7, 1952. 
following statements are pairwise equivalent:

(1) $A$ and $B$ are uniformly separable.

(2) If $\alpha$ is a vicinity of $X$, then $A-(A \cap B) \alpha$ and $B-(A \cap B) \alpha$ are uniformly separated.

(3) If $\alpha$ is a vicinity of $X$, then $A-(A \cap B) \alpha$ and $B$ are uniformly separated.

(4) If $\alpha$ is a vicinity of $X$, then there exists a vicinity $\beta$ of $X$ such that $A \beta \cap B \beta \subset(A \cap B) \alpha$.

Proof. It is clear that (1) is equivalent to (2).

Assume (2). We prove (3). Let $\alpha$ be a vicinity of $X$. Choose a vicinity $\beta$ of $X$ such that $\beta^{2} C \alpha$. Let $C=A \cap B$. Since $A-C \alpha$ is uniformly separated from both $B-C \beta$ and $C \beta$, and since $B C(B-C \beta)$ $\cup C \beta$, the conclusion follows.

Assume (3). We prove (4). Let $\alpha$ be a vicinity of $X$. Choose a vicinity $\gamma$ of $X$ such that $\gamma^{2} C \alpha$. There exists a vicinity $\beta$ of $X$ such that $\beta C \gamma$ and $(A-C \gamma) \beta \cap B \beta=\varnothing$ where $C=A \cap B$. It follows that $(A \beta-C \gamma \beta) \cap B \beta=\varnothing$ whence $A \beta \cap B \beta C C \gamma \beta \subset C \alpha$.

Assume (4). We prove (2). Let $\alpha$ be a vicinity of $X$. Choose a vicinity $\beta$ of $X$ such that $\beta^{2} C \alpha$. There exists a symmetric vicinity $\gamma$ of $X$ such that $\gamma \subset \beta$ and $A \gamma \cap B \gamma \subset C \beta$ where $C=A \cap B$. Hence $(A \gamma-C \beta)$ $\cap(B \gamma-C \beta)=\varnothing$. It follows that $(A-C \beta \gamma) \gamma \cap(B-C \beta \gamma) \gamma=\varnothing$. Thus $(A-C \alpha) \gamma \cap(B-C \alpha) \gamma=\varnothing$ whence $A-C \alpha$ and $B-C \alpha$ are uniformly separated.

Theorem 2. Let $X$ be a uniform space, let $Y$ be a dense subset of $X$, and let $A, B \subset Y$. Then $A$ and $B$ are uniformly \{separated \{separable in $Y$ if and only if $A$ and $B$ are uniformly separated $\}$ separable in $X$.

Proof. The first reading is clear. We prove the second reading.

Suppose $A$ and $B$ are uniformly separable in $X$. Let $\alpha$ be a vicinity of $Y$. Choose a vicinity $\beta$ of $X$ such that $\beta \cap(Y \times Y) \subset \alpha$. Define $\gamma=\beta \cap(Y \times Y)$ and $C=A \cap B$. Now $A-C \beta$ and $B-C \beta$ are uniformly separated in $X ; A-C \gamma=(A-C \beta) \cap Y$ and $B-C \gamma=(B-C \beta) \cap Y$ are uniformly separated in $X$ and therefore in $Y ; A-C \alpha$ and $B-C \alpha$ are uniformly separated in $Y$. Hence $A$ and $B$ are uniformly separable in $Y$.

Suppose $A$ and $B$ are uniformly separable in $Y$. Let $\alpha$ be an index of $X$. Define $\beta=\alpha \cap(Y \times Y)$ and $C=A \cap B$. Now $A-C \beta$ and $B-C \beta$ are uniformly separated in $Y$ and therefore in $X ; A-C \alpha$ and $B-C \alpha$ are uniformly separated in $X$. Hence $A$ and $B$ are uniformly separable in $X$. 
Theorem 3. Let $X$ be a uniform space and let $A, B \subset X$. Then:

(1) If $A$ and $B$ are uniformly separable, then $\overline{A \cap B}=\bar{A} \cap \bar{B}$.

(2) If $A$ or $B$ is conditionally compact in $X$ (in particular, if $X$ is compact) and if $Y$ is a dense subset of $X$ such that $A \cup B \subset Y$, then $\overline{A \cap B}=\bar{A} \cap \bar{B}$ (closures in $X$ ) if and only if $A$ and $B$ are uniformly separable in $Y$.

Proof. (1) Let $U$ be the uniformity of $X$. By Theorem $1, \bar{A} \cap \bar{B}$ $=\bigcap_{\alpha \in v} A \alpha \cap \bigcap_{\alpha \in v} B \alpha=\bigcap_{\alpha \in v} A \alpha \cap B \alpha \subset \bigcap_{\alpha \in V}(A \cap B) \alpha=\overline{A \cap B} \subset \bar{A}$ $\cap \bar{B}$.

(2) By Theorem 2 it is enough to show (2) for $Y=X$. Let $Y=X$. The sufficiency follows from (1). We prove the necessity. Let $\alpha$ be a vicinity of $X$. By Theorem 1 it is enough to show there exists a vicinity $\beta$ of $X$ such that $A \beta \cap B \beta C(A \cap B) \alpha$. Choose a vicinity $\gamma$ of $X$ such that $\gamma^{2} \subset \alpha$. Now $\bar{A}$ and $\bar{B}$ are uniformly separable and there exists a vicinity $\beta$ of $X$ such that $\bar{A} \beta \cap \bar{B} \beta \subset(\bar{A} \cap \bar{B}) \gamma$. Hence $A \beta \cap B \beta$ $\subset \bar{A} \beta \cap \bar{B} \beta \subset(\bar{A} \cap \bar{B}) \gamma=(\overline{A \cap B}) \gamma \subset(A \cap B) \gamma^{2} \subset(A \cap B) \alpha$ and $A \beta \cap B \beta$ $\subset(A \cap B) \alpha$.

Corollary 1. Let $X$ be a uniformizable space and let $A, B \subset X$. Then:

(1) If $A$ and $B$ are continuously separable, then $\overline{A \cap B}=\bar{A} \cap \bar{B}$.

(2) If $A$ or $B$ is conditionally compact (in particular, if $X$ is compact), then $\overline{A \cap B}=\bar{A} \cap \bar{B}$ if and only if $A$ and $B$ are continuously separable.

CoROllary 2. Let $X$ be a uniformizable space and for each $E \subset X$ let $E^{*}$ be the closure of $E$ in $\beta X$. Then:

(1) If $A, B \subset X$, then $(A \cap B)^{*}=A^{*} \cap B^{*}$ if and only if $A$ and $B$ are continuously separable in $X$.

(2) If $A, B \subset X$, then $A^{*} \cap B^{*}=\varnothing$ if and only if $A$ and $B$ are continuously separated in $X$.

(3) If $A$ is a compact subset of $X$ and if $B$ is a closed subset of $X$, then $(A \cap B)^{*}=A^{*} \cap B^{*}=A \cap B^{*}$.

(4) If $A$ is a compact subset of $X$, if $B$ is a closed subset of $X$, and if $A \cap B=\varnothing$, then $A^{*} \cap B^{*}=A \cap B^{*}=\varnothing$.

(5) The following statements are pairwise equivalent:

(i) $X$ is normal.

(ii) If $A$ and $B$ are closed subsets of $X$, then $(A \cap B)^{*}=A^{*} \cap B^{*}$.

(iii) If $A$ and $B$ are closed subsets of $X$ such that $A \cap B=\varnothing$, then $A^{*} \cap B^{*}=\varnothing$.

Statement (3) of Corollary 2 was proved in [2] under the additional assumption that $X$ is locally compact. For (5) compare [3]. 


\section{BIBLIOGRAPHY}

1. M. Katětov, On real-valued functions in topological spaces, Fund. Math. vol. 38 (1951) pp. 85-91.

2. A. D. Wallace, Extensional invariance, Duke Math. J. vol. 70 (1951) pp. 97102.

3. Henry Wallman, Lattices and topological spaces, Ann. of Math. vol. 39 (1938) pp. 112-126.

University of Pennsylvania 